\title{
Erratum to: Large intragenic deletion of CDC73 (exons 4-10) in a three-generation hyperparathyroidism-jaw tumor (HPT-JT) syndrome family
}

Vito Guarnieri ${ }^{1 *}$, Raewyn M. Seaberg ${ }^{2}$, Catherine Kelly ${ }^{3,4}$, M. Jean Davidson ${ }^{5}$, Simon Raphael ${ }^{6}$, Andrew Y. Shuen ${ }^{7}$, Filomena Baorda' ${ }^{1}$, Orazio Palumbo ${ }^{1}$, Alfredo Scillitani ${ }^{8}$, Geoffrey N. Hendy ${ }^{9,10}$ and David E.C. Cole ${ }^{7}$

\section{Erratum}

Following publication of the original article [1], the authors identified the following errors in the scientific content:

p.4, para. 3: "(1 tablet/10 mL RIPA)" should read "(1 tablet/10 mL RIPA buffer)".

p.4, para. 4: "II-1" should read "II-2".

Minor mistakes were also identified in the table on page 6, and in the Abbreviations section on page 7.

The original article has been corrected.

\begin{abstract}
Author details
${ }^{1}$ Medical Genetics, IRCCS Casa Sollievo della Sofferenza Hospital, San Giovanni Rotondo, Italy. ${ }^{2}$ Department of Otolaryngology - Head and Neck Surgery, University of Toronto, Toronto, ON, Canada. ${ }^{3}$ Department of Medicine, University of Toronto, Toronto, ON, Canada. ${ }^{4}$ Division of Endocrinology, Women's College Hospital, Toronto, ON, Canada. ${ }^{5}$ Department of Otolaryngology, Head \& Neck Surgery, Sunnybrook Health Sciences Centre, Toronto, ON, Canada. ${ }^{6}$ Department of Anatomic Pathology, Sunnybrook Health Sciences Centre, Toronto, ON, Canada. ${ }^{7}$ Departments of Laboratory Medicine and Pathobiology, Medicine and Genetics, University of Toronto, Toronto, ON, Canada. ${ }^{8}$ Endocrinology, IRCCS Casa Sollievo della Sofferenza Hospital, San Giovanni Rotondo, Italy. ${ }^{9}$ Metabolic Disorders and Complications, McGill University Health Centre-Research Institute, Montreal, QC, Canada. ${ }^{10}$ Departments of Medicine, Physiology and Human Genetics, McGill University, Montreal, QC, Canada.
\end{abstract}

Received: 7 September 2017 Accepted: 7 September 2017

Published online: 13 September 2017

\section{Reference}

1. Guarnieri V, Seaberg RM, Kelly C, Jean Davidson M, Raphael S, Shuen AY,

Baorda F, Palumbo O, Scillitani A, Hendy GN, Cole DEC. Large intragenic deletion of CDC73 (exons 4-10) in a three-generation hyperparathyroidism-jaw tumor (HPT-JT) syndrome family. BMC Med Genet. 2017;18:83. doi:10.1186/s12881-017-0445-0.

\footnotetext{
* Correspondence: v.guarnieri@operapadrepio.it

1 Medical Genetics, IRCCS Casa Sollievo della Sofferenza Hospital, San

Giovanni Rotondo, Italy
} 